Kompass

Pneumologie

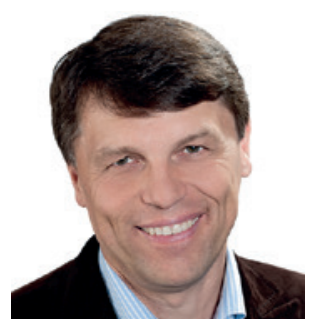

\section{Manfred Wagner}

Klinikum Nürnberg, Medizinische Klinik 3 - Pneumologie, Universitätsklinik der Paracelsus Medizinischen Privatuniversität, Nürnberg, Deutschland

\title{
Spotlights der pneumologischen Endoskopie
}

Dank des technischen Fortschrittes haben sowohl die Bronchoskopie als auch die internistische Thorakoskopie in den letzten Jahren einen kräftigen Schub erhalten. Durch verfeinerte Applikationswege haben altbewährte Methoden wie Kryotherapie, Laser- oder mechanische Biopsieverfahren neue Einsatzbereiche erlangt. Die Bronchoskopie kann in manchen Fällen mit Implantaten wie Stents oder Ventilen bei geeigneten Patienten mit Fisteln, zentralen Atemwegsstenosen oder schwerem Lungenemphysem ein Ersatz für thoraxchirurgische Eingriffe mit meist geringerem Trauma und gleichwertigen Erfolgsaussichten sein. Eingebrachte Implantate können in der Regel ohne großes Risiko wieder entfernt werden. Damit wurde die Bronchoskopie in der Diagnostik und in der Therapie von Krankheitsbildern unverzichtbar, in denen sie früher als nicht hilfreich oder gar als kontraindiziert galt. Im Fokus dieser Ausgabe steht der Einsatz dieser Methoden in Bereichen, die dadurch neue Perspektiven erfahren haben. In der Übersichtsarbeit von Kunyan Sun und Kollegen werden die Möglichkeiten und Grenzen in der Therapie der narbigen zentralen Atemwegsstenosen kritisch ausgelotet. Es können positive Prädiktoren für eine bronchoskopische Intervention mit oder ohne Stentimplantation definiert werden, anhand derer nach der nahezu regelhaft eintretenden schnellen Besserung auch langfristig ein guter Therapieerfolg vorhergesagt werden kann.

Wenn bei postoperativen bronchopleuralen Fisteln ein erneuter chirurgischer Eingriff nicht möglich ist, zeigen sich die positiven Effekte, aber auch Limitationen von konventionellen Stents und anderen Implantaten. Um die Einsatzbereiche dieser sicheren und meist reversiblen Maßnahmen zu erweitern, wird an neuen Technologien wie bioresorbierbaren Materialien und individuellen Maßanfertigungen mit einem 3D-Drucker gearbeitet. Hierbei sind jedoch neben den medizinischen auch die juristischen Aspekte zu berücksichtigen.

Die endoskopische Lungenvolumenreduktion beim schweren Emphysem ist seit vielen Jahren etabliert. Verbesserungen der Lebensqualität, der Leistungsparameter und der Überlebenszeit sind gut belegt. Mit der Messung metabolischer Parameter sollen weitere Kriterien ermittelt werden, mit denen der Behandlungserfolg optimiert werden kann.

Die internistische Thorakoskopie konkurriert in vielen Bereichen mit der chirurgischen videoassistierten Thorakoskopie (VATS). Der Aufwand ist jedoch wesentlich geringer. Neu ist der Einsatz der Kryothera- 
pie in der internistischen Thorakoskopie beim Pleuraempyem, das in diesem Fall damit weniger invasiv erfolgreich therapiert werden kann.

Bei Lungenrundherden ist die Bronchoskopie oft eine unverzichtbare Methode, jedoch vor allem bei kleinen Befunden in ungünstiger Lage nicht immer von hoher Treffsicherheit gekrönt. Neue Entwicklungen wie ultradünne Bronchoskope, endobronchialer Ultraschall mit radiären Minisonden (rEBUS), sowie aufwändigere Methoden wie die elektromagnetische Navigation (EMN) und künftig möglicherweise die robotergestützte Bronchoskopie versprechen Verbesserungen.

Abgerundet wird der Fokusbereich dieses Heftes durch eine Kasuistik, in der Daniela
Savi und Mitarbeitende von einer ungewöhnlichen allergischen bronchopulmonalen Aspergillose (ABPA) berichten. Die Bronchoskopie sollte auch hier möglichst frühzeitig eingesetzt werden, weil damit wegweisende diagnostische Erkenntnisse gewonnen und Krankheitsverläufe verkürzt werden können.

Diese Ausgabe gibt mit gelungenen, aber auch kritischen Beiträgen Einblicke in wichtige Einsatzbereiche der Bronchoskopie und Thorakoskopie. Die bisher schon rasanten Entwicklungen der Technik werden zukünftig fortschreiten und die Patientenversorgung weiter verbessern. Da neue Methoden nicht überall von Anfang an vorgehalten werden können, wird eine enge Vernetzung mit bronchoskopischen
Zentren immer wichtiger, damit diese wertvollen und sicheren Maßnahmen keinem Patienten vorenthalten bleiben.

Ich wünsche viel Freude und Erkenntnisgewinn bei der Lektüre und hoffe, dass damit ein Einblick in die faszinierende Welt der pneumologischen Endoskopie gegeben werden kann.

Ihr

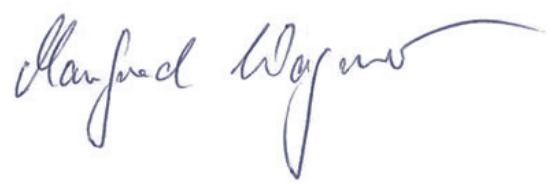

Dr. Manfred Wagner 